\title{
Ciência Viva-Promoting Marine Activities Around Lisbon: Self-Constructed Boats
}

\author{
Gonçalo Praça
}

Marine sports and activities for recreation, instruction, and tourism, among others, play a key role in increasing ocean literacy - the awareness of the mutual influence of ocean and human well-being. Recognising that marine leisure activities are relatively uncommon in Portugal and in Lisbon, compared with other activities and cities with similar geography, Ciência Viva proposed 'Kayaks to the River!', a series of workshops for building life-sized, usable kayaks, and designing activities with such boats to be part of a science festival in the river. The solution involves schools, clubs and citizens in the creation of a format for this initiative.

\section{Introduction}

Portugal is a coastal country famous for its mild weather and sunny beaches. The ocean plays a central role in its history, and its national mythologies and culture.

Compared to other countries and cities with similar geography, however, marine leisure activities are uncommon here. The Tejo estuary, bordering the capital Lisbon, one of the largest estuaries in Western Europe, is prepared for contemplation, not interaction. Public access to the water surface for recreational purposes is limited; current infrastructure and equipment are usually private and located in just a few locations.

\footnotetext{
G. Praça $(\varangle)$

Ciência Viva, Pavilhão Do Conhecimento, Lisbon, Portugal e-mail: gpraca@cienciaviva.pt
} 


\section{Ecosystem, Context and Challenge Addressed}

"Giving back the river Tejo to the city" is a recurrent theme in the governance of the city, frequently picked up by researchers, the tourism industry, pundits, and policymakers. The public has asked for access to the river, for instance in proposals for marine infrastructures and services regularly submitted to municipal participatory budgets. Urban regeneration projects for parts of the city, like Parque das Nações, the neighbourhood surrounding Ciência Viva's headquarters has explicitly included-at least in theory-the development of recreational activities in the river.

Ciência Viva, the Portuguese agency for scientific culture, has been involved in EU and national projects to develop "ocean literacy," i.e., raising awareness of the mutual influence of the ocean and human lives. Most of these projects, often directed to young participants, rest on top-down public engagement methodologies and goals: informal education with limited dialog and interaction between the public and experts, basic consultation devices and occasional hands on activities, showing experiments with citizen science and maker activities. These initiatives seldom propose direct interaction with maritime environments.

\subsection{Challenge}

In Portugal, activities in the ocean or in a big river like Tejo still tend to be considered either elitist, accessible to the rich; or as dangerous, deadly and connoted with poor, uneducated people. However, the idea of nurturing a "marine culture" has permeated policy agendas at the national, city and neighbourhood level. In Lisbon, for instance, historically, the Port Authority (a publicly owned limited company) rules access to the river. New regulation is coming into force that transfers some of the ruling power to the Municipality, or even to the neighbourhood, pursuing an agenda of urban sustainability in terms of leisure, tourism, mobility, spatial planning. This agenda has the scope of increasing sports practice, raising environmental awareness and fostering civic care for public spaces.

This tension was noted in projects for "ocean literacy" in which Ciência Viva has been involved. Marine sports and activities for recreation, fun, instruction or tourism are crucial to increase "ocean literacy." However, to obtain any meaningful impact in engaging the public with ocean literacy, marine leisure activities must be widely and regularly practiced and be easily accessible — which does not happen in Portugal.

Members of Ciencia Viva team wanted to address this gap, and the lab set itself a challenge that resonates with these ideas: how to devise interesting, mobilising, safe and accessible experiences in Tejo, especially in the section of the river close to Pavilion of Knowledge. 


\section{The Co-Creation Journey}

\subsection{Context Analysis}

Analysis of the context rested on desk- and field research. Desk research focused on the considerable body of research and "gray" literature on recreational boating and water-based sports in Portugal and in Lisbon are, including statistics, reports, dissertations, papers, propaganda, etc. mainly from researchers in geography and urban planning; tourism; economy and innovation; cultural heritage; and sports.

Field research included interviews with key stakeholders previously identified, in particular individuals from professional networks and citizens familiar with maritime activities in the area of Lisbon, Portugal or other countries. A crucial dimension of fieldwork was the observation of the actual "design" of the river and its uses in the area of Lisbon, documenting it with photos, short videos and field notes. This included active participation in activities to address problems pertinent to the challenge.

All the material gathered was organised with basic qualitative coding techniques, identifying themes and trends, mapping stakeholders, and comparing and merging different SWOT analysis.

\subsection{Problem Framing}

To revise and refine the initial challenge of engaging the public with the river, the root problem of restricted active use of the river was untangled. Themes emerging in this phase revealed two major dimensions of the problem: limited physical access to water (due to inappropriate infrastructure and real or perceived costs); and a hazy yet meaningful "cultural" resistance to water-based activities.

Two main tools helped synthesising this information:

1. A SWOT analysis of leisure water activities in Portugal, in Lisbon and in the specific neighborhood, making visible dimensions of the problem that the co-lab could address meaningfully; and

2. A detailed stakeholder map with a clear idea of the interests, needs, skills and relationships between current and potential stakeholders. This helped reframe the initial challenge to make it more workable and related to specific people and groups.

The initial challenge was substantially reframed during a workshop with core stakeholders. At this stage, the challenge was "How can we show that the river in this part of the city is interesting, accessible and safe-but that it needs attention from authorities for its fruition."

Finally, the internal team further refined this challenge with the participants of an idea generation workshop. The challenge that eventually guided the idea generation 
was "What interesting, mobilising, safe and accessible experiences could our co-lab create in the river in this part of the city?"

Reframing the problem, in short, meant turning it from a general concern for promoting "ocean literacy" into something that should consider the specific needs and aspirations identified in fieldwork. The ambition was now reasonably clear: to show that the river is there, and it should be used, even if measures are needed; inviting people to literally get themselves immersed in the water; and to deploy engaging examples, instead of repeating worn-out publicity campaigns.

\subsection{Envisioning Solutions}

Stakeholders and internal team members imagined possible solutions from the very start of the project: it seemed natural to think of a problem by thinking of solutions, inspiring internal discussions that lead to both different perspectives on the challenge and to other ideas, one of which would later resurface during more formal ideation sessions.

In the second workshop, 13 participants discussed and wrote ideas on cards answering the question: "What interesting, mobilising, safe and accessible experiences could theco-lab create in the river in this part of the city?" The group categorised solution cards in a matrix with quadrants representing the challenge (access to sea/river; mobilisation; safety; interest). A trend started to emerge, with most ideas placed in the areas titled "interest" and "mobilisation."

The group agreed that the more promising cluster in terms of benefits was the set of solutions that involved immersive experience in the river as it is now, without complex and expensive interventions. This has been taken as an invitation to develop a solution addressing the need to show and communicate that the river is offering various possibilities already interesting and accessible. In short, to devise activities in the river capable of mobilising diverse publics, using available infrastructure and equipment, while drawing the attention of authorities for improving this equipment and infrastructure.

Another workshop with the participation of around 150 citizens (families, in particular) explored the question "Why would I want to attend an event in the river? What activities in it would move me?" to identify recurring and similar ideas and concepts most fitting to users' needs.

The solution resulting from these meetings was thus devised as an annual workshop for constructing life-sized, usable watercrafts. The boats should be exhibited in the water, in different uses, contextualised by a multidisciplinary festival devoted to the river/sea. 


\subsection{Developing and Prototyping}

Just as the solution was emerging, the internal team came across an interesting, but barely known project, Abraçar o Vento ("Embracing the wind") [1]. The project stems from a partnership with Marquesa de Alorna, ${ }^{1}$ a lower secondary school in Lisbon, the nautical club Boa-Esperança, ${ }^{2}$ and GIRA, ${ }^{3}$ a nonprofit association for the rehabilitation of adult mental disease patients [2-4]. Abraçar o Vento aims at fostering informal peer-to-peer learning to raise awareness about water sports and environmental protection, and promote social inclusion-all this around a DIY kayak workshop hosted by the school, in which students, adult patients, a carpenter and a "mentor" build complete kayaks based on an open-access template.

This encounter had profound implications for the solution. Indeed, development and prototyping phases were devoted to understanding how to generalise, expand and innovate from this learning experiment in Ciência Viva's own workshop-withfestival.

Discussions involving partners and stakeholders like the municipality, associations related to amateur boat-building and nautical leisure activities, teachers, and a video producer of a documentary on social innovation guided prototyping of an annual festival based on the construction of DIY kayaks built upon the experiment of the school running for the (interrupted) school year of 2019-2020. In practice, developing and prototyping included partial immersion in key moments of kayak construction with students and adult patients in a real setting; this also involved stakeholders in one workshop, and during later incursions to the school.

The team deliberately looked for simple visual tools, accessible to the internal team, stakeholders, and other staff from Ciência Viva to develop the solution: mind maps, storyboards, user journey maps and, in later stages of prototyping, digital mockups, and service blueprint templates. For the work on the tools and discussions of emerging results, the working group used material and virtual feedback walls, all organised around clear dimensions: like/would like/issues and doubts/suggestions, needs/requirements, goals, etc.

Developing and prototyping brought some light on aspects of the learning module, especially four main topics:

- Motivation for participation (final users and stakeholders);

- Identification of contents, material and the network needed to put the initiative in practice;

- Resources needed for the learning process (templates, tutorials, live training);

- Adaptability of the module (different versions, templates, adaptation to various situations).

Despite all restrictions due to the COVID-19 outbreak, workshops with stakeholders and colleagues offered important guidance for the service that was being

\footnotetext{
1 agmarquesadealorna.wixsite.com/marquesadealorna.

2 www.ncbe.pt/index.html.

${ }^{3}$ www.gira.org.pt/.
} 
prototyped. Main instructions following from these workshops include: allow and stimulate peer-to-peer-learning, learning by doing with others; use peer-to-peerengagement, i.e. showing, motivating, changing attitudes by example; and trying and failing - instead of the successful reproduction of existing safe models.

Initially, the solution focused on a festival that should populate the river with usable kayaks, built by teams according to a tested template. The contact with the school, and subsequent discussions with other partners, led to a much stronger emphasis on the peer-to-peer learning processes that should occur during the co-design and construction of kayaks. This, in turn, should translate into the design of contents of the festival itself as well.

\subsection{The Role of Policies and Policymaker Engagement}

Initial reports of the lab with policymakers - the municipality and the neighbourhood governments-piggybacked on the collaboration and personal networks between local policymakers and Ciência Viva. Perhaps for this reason, early engagement was straightforward; policymakers were open to meetings and expressed interest in collaborating, gave insightful information and offered to help in activities like dissemination or events in the neighbourhood.

Furthermore, it helped the challenge and, later, the developed solution, fit the agendas of different departments of the Municipality (Environment, Sports, Sea economy) of "giving back the river to the people." Local policymakers consider Ciência Viva a well-regarded influencer and expressed their trust in it to help to raise public interest in these activities, and to help lobby for improving public access to the river.

Once the solution became clearer, it was possible to raise the interest of a team of the municipality and national ministries involved in the organisation of a major event in Lisbon: The UN Ocean Conference that would occur within the period of the pilot. While the initiatives were cancelled due to the pandemic, the team discussed with them how to include the solution in the agenda of this event, as a pilot version of the workshop-festival. It was also considered how such an initiative could become part of a national agenda for ocean literacy.

Involving policymakers into actual co-creation turned out to be more complicated: a lack of participation in events and the support of concrete ideas for initiatives hindered their active involvement.

On the other hand, they were important to validate initial stages of the prototype: when addressing the solution in workshops or individual interviews, policymakers from the City and neighbourhood offered important suggestions to make it more realistic and implementable considering specific financial and organisational constraints that they know well.

A specific challenge related to the engagement of policymakers was ensuring a safe and comfortable space for them during the journey. In Lisbon, there are just a few actors in the fields of water leisure activities and ocean literacy; and they carry with 
them a history of unaddressed past and present issues. All these dimensions emerged during the journey, not without some tensions, in exchanges between policymakers and other stakeholders.

\section{Experimentation: Output, Transformations, Outcomes}

Ciência Viva proposes to develop an annual festival devoted to the DIY design, adaptation and/or construction of real size kayaks usable in rivers or similar conditions. The initiative starts with a call directed to the school community in Greater Lisbon, in particular teachers, students and staff involved with Ciência Viva. Participants will contribute by building collaborations for the co-construction of kayaks, and document their experiences to share them with others.

Looking at this concept as a service, it encompasses:

- An online learning and engagement module focused on boat design, building and co-creation skills, dissemination;

- Show and tell, "make happen" activities: demos and workshops for the public about DIY boat building and related skills;

- And the dissemination of awareness and advocacy initiatives for engagement in creative citizenship in the river.

The learning module should be used — and developed—in the school year, while the festival would be an annual event before the summer break.

An important value of the solution is embedding the ideals of co-creation deeply within the participants' experience; they will be challenged to create and develop contents for all components of the initiative-learning processes, documenting and dissemination and engagement.

Participants should work in multi-stakeholder teams, exploring diversity in gender, age and skills, recruited in their schools and their creative ecosystem, like makerspaces, local associations or even businesses. They will be invited to add as many creative "layers" as they wish, developing their kayaks in terms of design, materials, artistic dimensions, uses, and activities for the festival.

This work will be fully documented and made available on the project's site to feed the learning module itself and foster future participation in the initiative. Ultimately, participants will be encouraged to develop, complement or revise contents offered in the site (e.g., manuals, tutorials), with their ideas and creative work (including "making of," things that did not work), much in the spirit of recent open DIY innovation platforms like wikifactory.com or www.scopesdf.org.

The technical core of the solution is the online peer-to-peer learning and engagement module, planned to have five main sections:

- Kayaks to the river! Presenting the initiative; how to participate; steps of the creative process; submitting proposals; rules; calendar. 
- Mission A "manifesto" for the active and creative citizenship in the river, and how it can translate into the participant's missions: design, transform, build your own kayak, and co-create the final event.

- Resources Set of resources to help design, building and transforming kayaks, including specific tools for (remote) creative work. Continuously updated by participants, a wiki for DIY kayak construction.

- Galleries Photos and videos of/by participants, about their creative journeys.

- Help Ongoing FAQ, fed by issues raised by participants. Contacts, including contacts for "mentors" selected from relevant stakeholders of the lab.

The online module represents a typical creative journey: participants get familiar and interested in the initiative to then form teams and define specific goals reflecting on the values stated in the initiative's manifesto, and inspired by examples offered on the site. They choose formats, materials and tools to present a summary of the idea and composition of the team. They then develop their proposals-in sketches, $\mathrm{CAD}$, to scale or real-size physical prototypes, etc.- - with resources (tools, tutorials, templates, etc.) available. "Mentors" selected by a pool of the lab's stakeholders are available along the process. Participants will document the development of their proposals, to feed both an ongoing wiki for DIY kayak construction and the multimedia galleries showing their creative journeys. Proposals are submitted and reviewed by lab's stakeholders and mentors, who will provide relevant feedback. Participants then work on their final models of the kayaks. Two not mutually exclusive scenarios for this work can be anticipated: (1) the models, and related activities designed by the participants will provide the contents of a weekend event in the river; or, worst-case, pandemic scenario (2) the models and related documentation will feed an online event.

\subsection{Transformations Triggered}

Before SISCODE, co-creation in Ciência Viva was an ideal that some of its staff were more or less familiar with; and selected aspects of it, rather than complete processes, were used in single tasks of several RRI projects. It is still premature to speak of permanent transformations within the organisation fed by the spirit of co-creation, and changes induced by the pandemic added a layer of uncertainty in this regard. Still, it is fair to say that Ciência Viva started developing more explicit, deliberate, reflexive co-creation processes during the pilot—and thanks to it.

The metaphor of the co-creation journey, and the methodologies and tools developed in the process were presented to staff members not familiar with SISCODE in informal environments to foster their spread beyond the project. These methodologies and tools were also shared and used in the following situations:

- Co-creation for Science Centers and Museums: training workshop using the metaphor of co-creation journey, and selected canvases from the toolbox; 
- A class in the MSc degree in science communication at Universidade Nova de Lisboa;

- Idea generation for 2020s programme: day-long meeting(s) of Ciência Viva's Outreach unit;

- Co-creating engagement strategies for a Science Center with "hot topics": trial co-creation session with Ciência Viva's Outreach and Education units;

- Developing a programme for a national event of Ciência Viva: collaboration with teachers nationwide.

During the lockdown, members of Ciência Viva staff external to the lab joined a couple of the online workshops; for some of them, this was the first contact with cocreation methodologies. Most Ciência Viva's participants in the activities mentioned here expressed their wish of developing these skills with more formal training that should be provided by the organisation.

\section{Lessons Learnt and Reflections}

The concept of "hermeneutic circle" is particularly illuminating to describe the learning process during this experiment [5]. This refers to the idea of continually moving from smaller component parts to a larger unit, and back, in order to understand the meaning of both-the individual parts and the system as a whole. In this specific case, it becomes visible that the single components and phases of the journey have no "meaning" in themselves; the sense of the journey does not derive merely from adding up phases one after the other; rather, the importance of the journey and of the individual phases from a learning point of view can only be grasped from the complete process.

A basic implication of this idea is that the lab (and colleagues not necessarily involved in SISCODE) reached the end of the experiment much more aware of the specific skills and knowledge that should still be developed to design and carry out co-creation processes in future projects.

But the concept of the hermeneutic circle also helps have a more explicit understanding of the iterative nature of co-creation, and about the specific experiment of Ciência Viva. For instance, the team only understood the generative power of prototyping - i.e., how engaging with actual objects and products can accelerate new ideas and new connections between different people — close to the end of the journey; but to understand this it was necessary to have abstract discussions before. Also, it is only towards the end of the process that issues and ideas that emerged early in the journey could be taken into account.

For instance, participants in the first co-creation sessions stated along these lines: "The best way to teach someone how to swim is throwing them to the water, pardon my French, but if you want to engage people with the river that's the only way to do it"; or this: "We shouldn't waste our time with more campaigns, we have to put the public to use to show other people how this is fun"; or: "We should all go to 
the river, a big parade, then people outside would see us, 'Ah, that looks fun, I want to do it!'". It was only after several trial and error phases that these ideas could be fully understood; it took the whole "circle" to make justice to the aspirations they reflected.

\section{References}

1. Abraçar o Vento. bit.ly/2CNEZFM. Last accessed 02 Oct 2020

2. GIRA. www.gira.org.pt/. Last accessed 02 Oct 2020

3. Marquesa de Alorna School. agmarquesadealorna.wixsite.com/marquesadealorna. Last accessed 02 Oct 2020

4. Nautical club Boa-Esperança. www.ncbe.pt/index.html. Last accessed 02 Oct 2020

5. University of Leiden. The hermeneutic circle. https://www.youtube.com/watch?v=zIEzc BBxs. Last accessed 29 Mar 2021

Open Access This chapter is licensed under the terms of the Creative Commons Attribution 4.0 International License (http://creativecommons.org/licenses/by/4.0/), which permits use, sharing, adaptation, distribution and reproduction in any medium or format, as long as you give appropriate credit to the original author(s) and the source, provide a link to the Creative Commons license and indicate if changes were made.

The images or other third party material in this chapter are included in the chapter's Creative Commons license, unless indicated otherwise in a credit line to the material. If material is not included in the chapter's Creative Commons license and your intended use is not permitted by statutory regulation or exceeds the permitted use, you will need to obtain permission directly from the copyright holder.

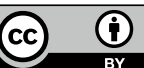

